\title{
The Development of the Thermal Object Intelligent Adaptive Identification Software Based on the Operation Data
}

\author{
Nan Xu ${ }^{\text {a }}$, Junlin Guo ${ }^{\text {b }}$, Shuhao Zhang ${ }^{c}$, Wenjie Zhao ${ }^{\mathrm{d}}$ \\ North China Electric Power University, \\ Baoding, China,

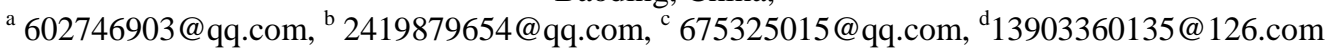

\begin{abstract}
In this paper, we introduce the principle and development of the thermal object intelligent adaptive identification software based on the operation data, and discuss the application of the genetic algorithm in system identification. We completed the design of graphics user interface with the help of GUIDE. Using the software for system identification, not only simplifies the identification process, but also can directly reflect the gap between the identification data with the experimental data. Then through a concrete example, we verify the usefulness of the software in the model identification of the thermal object, which is with good project application significance.
\end{abstract}

Keywords-System identification; Genetic algorithm; Graphics user interface; GUIDE

\section{INTRODUCTION}

There are a lot of control object in the actual project, whose characteristics need to be described by establishing a mathematical model. In the case of incomplete prior knowledge, we need to use identification theory to extract information from input and output data in the system and construct the system model ${ }^{[1]}$. Identification theory is on intimate terms with the practice. Because of the complex diversity of the actual model, we need a large number of experimental studies, then we may solve the practical modeling problems ${ }^{[2]}$.

In this paper, our research is based on the thermal object in the power plant. Thermal object is a complex object, which is described by higher order nonlinear differential equations. In practice, the transfer function is usually used to describe the dynamic characteristics ${ }^{[3]}$. Due to the complexity of the thermal object, the mathematical model of the transfer function can't be accurately obtained. We usually use the method of system identification to obtain the important parameters to characterize the thermal object, and establish a mathematical model which can imitate its real behavior $^{[4]}$.

The design of GUI consists of two parts: the interface design and the preparation of the control procedures ${ }^{[5]}$. In this paper, with the help of the design tool of MATLAB GUI, we make the code with system identification function to associate with the GUI controls, and develop a thermal object intelligent adaptive identification software based on the operation data. Users do not have to write the code, as long as activate the GUI controls, and enter input and output data collected in the field, and set the relevant parameters in the software interface. Then the software can automatically identify and get an accurate system transfer function model as well as the response curve.

\section{THE PRINCIPLE OF IDENTIFICATION BASED ON GENETIC ALGORITHM}

Identification uses the error between the model output and the actual output to constantly correct the model parameters, and ultimately get the optimal process model through some kind of algorithm. There are many identification methods, such as frequency response method, impulse response method, least square method, intelligent algorithms ${ }^{[6]}$. In this paper, we adopt the intelligent identification algorithm based on genetic algorithm.

According to the model analysis of the thermal process, a mathematical model of one thermal process in the vicinity of the equilibrium point is expressed as follows:

$$
w(s)=\frac{y(s)}{u(s)}=\frac{k}{(T s+1)} e^{-\tau s}
$$

Where, $y$ and $u$, respectively, are the output and input of the actual object.

The true value of the parameter:

$$
\theta=[k T n \tau]^{T}
$$

The estimation value of the parameter:

$$
\theta^{*}=\left[k^{*} T^{*} n^{*} \tau^{*}\right]^{T}
$$

The principle of system identification based on genetic algorithm is as shown in Figure 1.

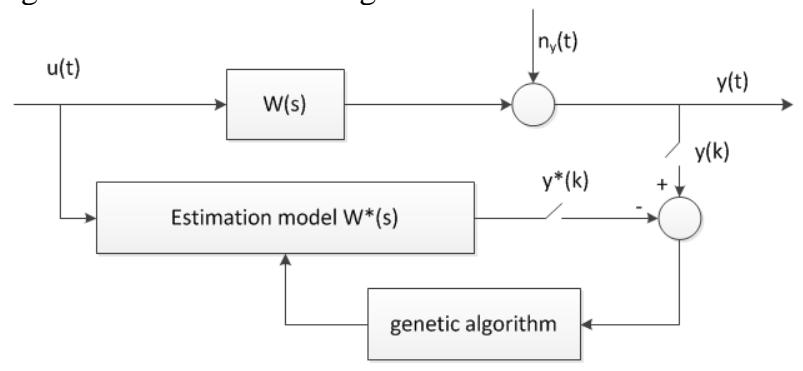

Fig. 1 Schematic diagram of system identification based on GA 
In figure $1, n_{y}(t)$ is the output noise, $y(t)$ is the actual continuous output with noise, $y(k)$ is the sample values of $y(t), y^{*}(k)$ is the sample values of estimating the model continuous output. $y^{*}(k)$ is essentially different from the discrete sequence $\left\{y^{*}(k)\right\}$ generated by recursion according to discrete input sequence $\{u(k)\}$. When programming, we adopt the continuous function of MATLAB simulation software, "lsim", to produce $y^{*}(k)$. The process of model identification is to find the optimal estimation parameters $\theta^{*}$, and to make the following minimum objective function value:

$$
J(k)=\sum^{n}\left[y(k)-y^{*}(k)\right]^{2}
$$

\section{DESIGN OF IDENTIFICATION SOFTWARE}

The software is a kind of identification tools based on the thermal object. According to the input and output data from EXCEL format data files and the specified model structure, it can use intelligent identification algorithm based on genetics to identify the various parameters of the model, and give the validation curve of identification results. The software mainly consists of the following three modules:

\section{A. The data reading module}

This module is the interface between MATLAB and EXCEL file. Its function is to read the actual process data from the EXCEL file and the settings of identification parameter in the interface.

Using 'get' function to read the input data. The syntax is:

$$
\text { get(handles.T;PropertyName) }
$$

Where, $\mathrm{T}$ is an identifier of data acquisition control, handles.T is the handles of this control, PropertyName represents the name of the control properties required to be acquired.

But the data extracted by the 'get' function is string, which need to call str2double function to convert a string into numerical values. The syntax is:

$$
\mathrm{X}=\text { str2double('str') }
$$

Where the str is the real or complex represented by a ASCII string, this call format convert a string into a numeric value.

\section{B. GA identification module}

This module is the computing center of the package. We get the process data by the data reading module to deal with the raw data, and use MATLAB GA toolbox to identify model parameters with genetic algorithms for thermal objects.

\section{The graphical user interface module}

This module is the interface of user and computer interaction. It provides a desktop operating environment with friendly interface for the user.

The software has six interfaces in total, respectively as follows:

(1)Selection of data time interface

The data used to identify can be produced through the step input disturbance test. So the data segment of the start-stop time of beginning and ending time for the test, as shown in figure 2.

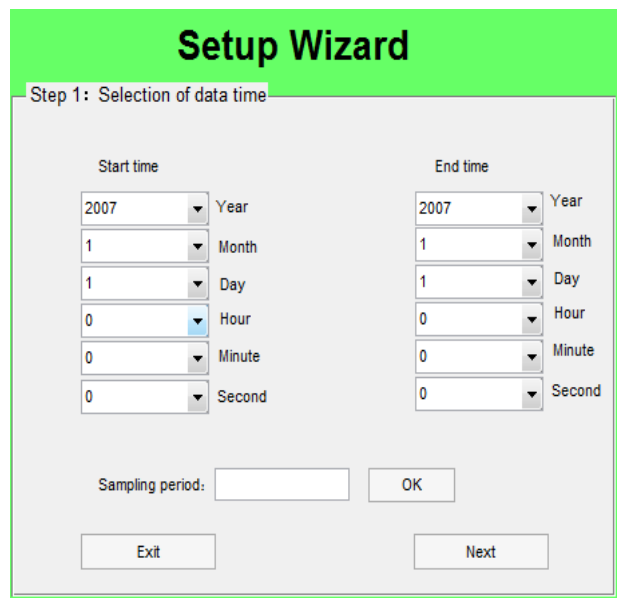

Fig. 2 selection of data time

(2)Selection of data path interface

This interface selects the original thermal-hydraulics EXCEL file used for identification. This file can be stored in a computer any of the specified directory, as figure 3:

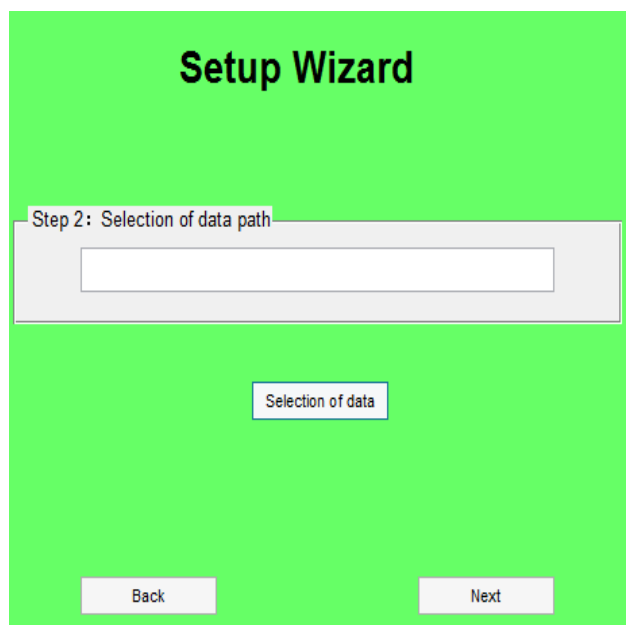

Fig. 3 selection of data path

(3)Selection of state variable interface

When the storage of data file path has been set, file variable names is shown in the man-machine interface of the drop-down menu in the automatic sequence, as figure 4 : 


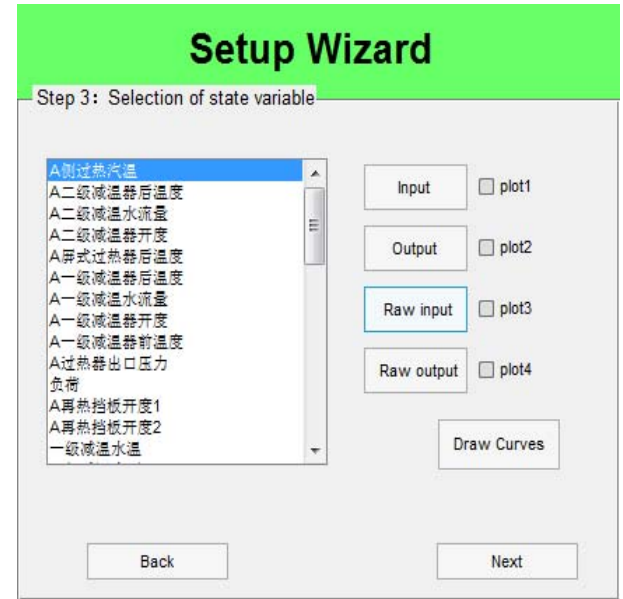

Fig. 4 selection of state variable

(4)Selection of model and parameter interface

According to the several kinds of common thermal process model structure of the preset, it selects object model structure which is consistent with identification through the drop-down menu, then sets up the upper and lower of the model parameters based on experience, as figure 5 .

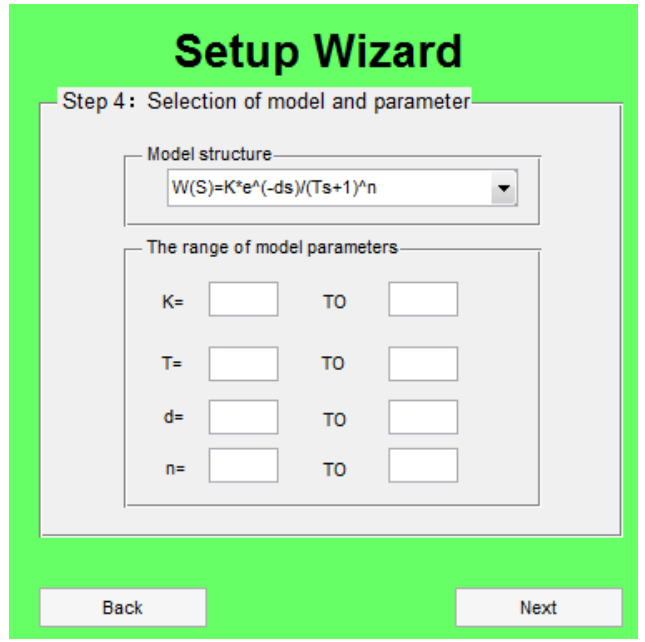

Fig. 5 selection of model and parameter

(5) Selection of parameter identification interface

This interface sets control parameters of the genetic algorithm. By clicking on the button 'identifying input' and "identification of output", it can complete the preprocessing of the identification data, as shown in figure 6.

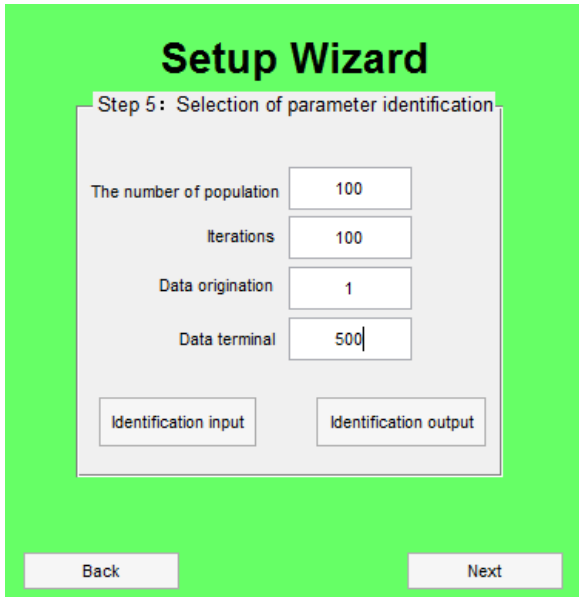

Fig. 6 selection of identification parameters

(6)Identification of implementation interface

Click on the button 'identification start', the system automatically identify the model parameters based on the genetic algorithm, according to the given conditions, such as input/output data. And then it shows the identification of model structure and the closeness between the practical process data, as shown in figure 7 .

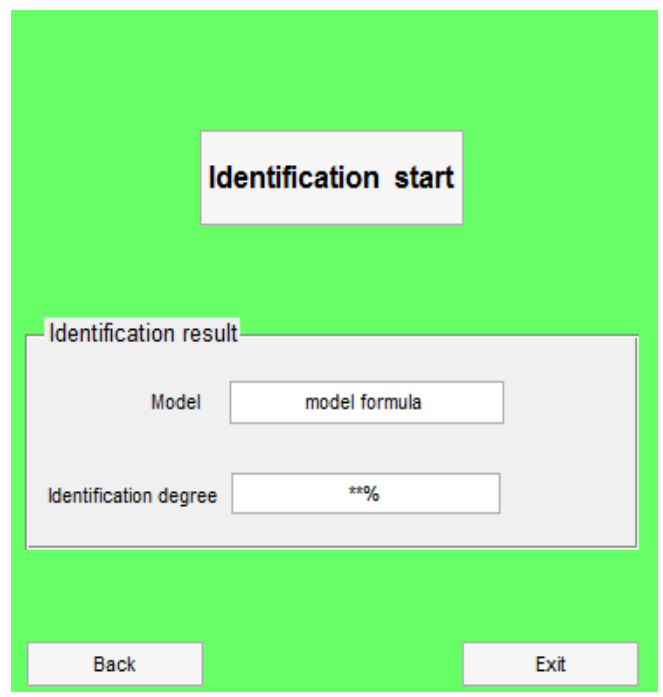

Fig. 7 identification results

\section{APPLICATION EXAMPLES}

Take power plant operation data for example, we choose water spray desuperheater as the thermal object for research. The input is desuperheating water flow, while the output is the leading steam temperature (temperature after desuperheater). According to the experience, the structure mode of the object can be described as follows:

$$
w(s)=\frac{k}{(T s+1)} e^{-\tau s}
$$

By looking at the test data curve can judge the effect of the experiment, then further determine the validity of the 
identification software. Identification result images as shown in figure 8.

The system transfer function model of the object is:

$$
w(s)=\frac{7.33}{(263 s+1)^{2}} e^{-224 s}
$$

Identification degree was $95.3258 \%$, so the identification effect is ideal.

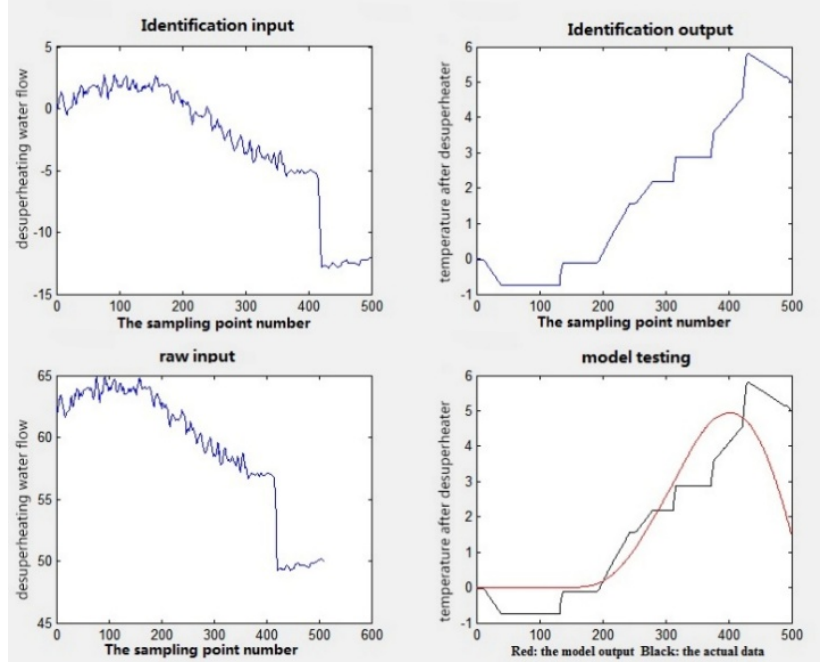

Fig. 8 identification image

\section{CONCLUSION}

As can be seen from the above, this identification software makes full use of the data visualization technology and supporting for dynamic system simulation of MATLAB, and implements the identification of thermal object model together with genetic toolbox flexible. The software adopts the open design framework which can constantly add more content, including the identification of special system and control problem based on model identification, etc. To get the result of identification, users only need to input the data instead of writing code. And it can compare with the actual data intuitively. Meanwhile, it can identify many times to find the most practical system transfer function. And is suitable for the practical application of engineering.

\section{ACKNOWLEDGEMENT}

This paper is supported by Natural Science Foundation of Hebei Province of China (No.F2014502059).

\section{REFERENCES}

[1] Lennart Ljung, Black box model from input output measurement [C] IEEE Instrumentation and Measurement Technology Conference1, B udapest, Hungary, 2001: 21-23.

[2] Ni, Biyi, and Xiao Deyun. The MATLAB simulation environment of system identification toolbox[J]. Journal of system simulation. 2006,18(6):1493-1496.

[3] Sun, Jianping, Tan Yue and Ma Huajie .Nonlinear identification algorithm and its application in the thermal object[J]. Journal of north China electric power university. 2005,32(5):32-34.

4] Mi, kesong, Yang Bo and Yang Jianmeng. Based on system identification of feed water control of $600 \mathrm{mw}$ supercritical thermal object modeling and simulation[J]. Applied Energy Technology. 2011,(9):26-29

[5] Yao, Xiufang, and Cui Songfei. Based on MATLAB GUI programming. Computer Knowledge and Technology. 2009,5(20):7767-7768.

[6] Liu, Changliang. Coal-fired power plant thermal process model identification based on genetic algorithm[J]. Proceedings of the CSEE. 2003,23(3): 170-174 